\title{
Inversion-symmetry protected chiral hinge states in stacks of doped quantum Hall layers
}

\author{
Sander H. Kooi, ${ }^{1}$ Guido van Miert, ${ }^{1}$ and Carmine Ortix ${ }^{1,2}$ \\ ${ }^{1}$ Institute for Theoretical Physics, Center for Extreme Matter and Emergent Phenomena, Utrecht University, \\ Princetonplein 5, 3584 CC Utrecht, Netherlands \\ ${ }^{2}$ Dipartimento di Fisica “E. R. Caianiello”, Universitá di Salerno, IT-84084 Fisciano, Italy
}

(Received 4 July 2018; published 3 December 2018)

\begin{abstract}
We prove the existence of higher-order topological insulators with protected chiral hinge modes in quasitwo-dimensional systems made out of coupled layers stacked in an inversion-symmetric manner. In particular, we show that a homogeneous external magnetic field slightly tilted away from the stacking direction drives alternating $p$ - and $n$-doped honeycomb sheets into a higher-order topological phase, characterized by a nontrivial three-dimensional $\mathbb{Z}_{2}$ invariant. We identify graphene, silicene, and phosphorene multilayers as potential material platforms for the experimental detection of this second-order topological insulating phase.
\end{abstract}

DOI: 10.1103/PhysRevB.98.245102

\section{INTRODUCTION}

A free-fermion symmetry protected topological (SPT) insulator is a quantum state of matter that cannot be adiabatically deformed to a trivial atomic insulator without either closing the insulating bulk band gap or breaking the protecting symmetry $[1,2]$. Its topological nature is reflected in the general appearance of gapless boundary modes in one dimension lower $[3,4]$. However, when the protecting symmetry is a crystalline symmetry the gapless boundary modes appear only on surfaces which are left invariant under the protecting symmetry operation [5,6]. Most importantly, these gapless boundary modes are "anomalous": on a single surface the number of fermion flavors explicitly violates the fermion doubling theorem [7] or stronger versions of it [8].

Very recently, it has been shown that point-group symmetries can stabilize insulating states of matter in bulk crystals with conventional gapped surfaces, but with gapless modes at the hinges connecting two surfaces related by the protecting crystalline symmetry [8-24]. For systems of spinless electrons (negligible spin-orbit coupling) the hinge modes are chiral. Hence, they represent anomalous one-dimensional (1D) modes - they cannot be encountered in any conventional 1D atomic chain-but now embedded in a three-dimensional crystal. These novel topological crystalline insulators, which have been dubbed higher-order topological insulators, have started to be classified in systems possessing different crystalline symmetries, including rotational and rotoinversion symmetries [9,10,20,22,25].

In inversion-symmetric crystals, higher-order topological insulators can also exist [18]. However, in this case, inversionsymmetry related surfaces are connected via two hinges, one of which will host a chiral gapless mode. This, in turn, gives rise to an additional modulo two ambiguity in the microscopic hinge location of the chiral modes, reminiscent of the ambiguity in the Fermi arcs connectivity of Weyl semimetals [26].

The aim of this article is to show that such an inversionsymmetry protected higher-order topological insulator can be in principle obtained in stacks of doped honeycomb layers (e.g., graphene, silicene, and phosphorene) subject to an external magnetic field tilted away from the stacking direction.

Two factors conspire to render this possible. First, the quantum Hall states in $p$ - and $n$-doped layers generally have opposite sign of the Hall conductance and hence are characterized by opposite Chern numbers $\mathcal{C}$ [27]. Second, a finite component of the magnetic field in the layer planes intrinsically breaks both the reflection symmetry in the stacking direction and the twofold rotation about the stacking direction, but still preserves the three-dimensional bulk inversion symmetry. We first give an intuitive argument for the existence of an inversion-symmetric higher-order topological insulator in stacks of Chern insulators with alternating $\mathcal{C}= \pm 1$ integer invariants, and show that this insulating phase can be derived from a parent mirror Chern insulator [28] by adding crystalline symmetry-breaking terms. Then, we introduce a corresponding minimal tight-binding model consisting of quantum anomalous Hall layer stacks [29], and verify its topological nature by computing the corresponding bulk $\mathbb{Z}_{2}$ invariant [25]. Finally, we perform a full Hofstadter [30] calculation in three dimensions [31] for honeycomb layers doped in an alternating fashion to show the existence of topologically protected chiral hinge states.

\section{EFFECTIVE SURFACE THEORY}

Let us start out with an effective low-energy approach for stacks of Chern insulating layers of alternating $\mathcal{C}= \pm 1$ integer topological invariant [cf. Fig. 1(a)]. At any edge perpendicular to the stacking direction, each Chern insulating layer is characterized by an anomalous chiral edge mode whose dispersion can be considered to be linear. For completely uncoupled layers, the effective surface Hamiltonian in the primitive two-layer surface unit cell then reads $\mathcal{H}_{0}=k_{x} \sigma_{z}$, where the Pauli matrix acts in the layer space and we explicitly considered a (010) surface. We next introduce an interlayer coupling between consecutive layers with a coupling strength, which, for simplicity, we assume to be real. The effective 

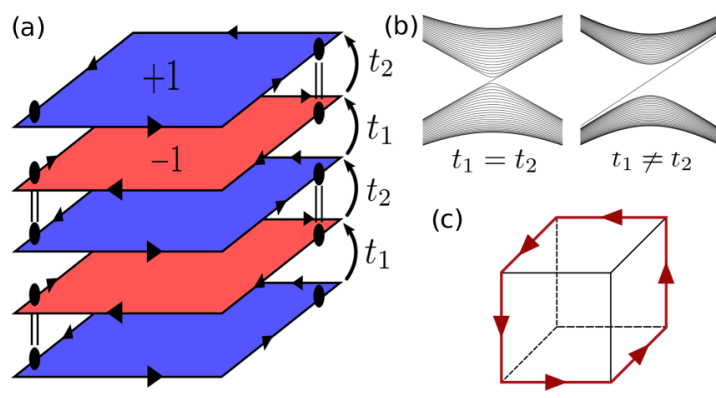

FIG. 1. (a) Sketch of stacked Chern insulators with alternating Chern numbers. We also indicate the effective inversion-symmetric coupling between the chiral edge states. (b) Corresponding surface energy spectrum for mirror symmetric couplings $\left(t_{1}=t_{2}\right)$ and inversion-symmetric couplings $\left(t_{1} \neq t_{2}\right)$. (c) Schematic figure of the inversion-symmetric hinge states in a cube geometry.

surface Hamiltonian is then modified accordingly to $\mathcal{H}_{S}=\mathcal{H}_{0}+t\left[1+\cos \left(k_{z}\right)\right] \sigma_{x}-t \sin \left(k_{z}\right) \sigma_{y} . \quad$ It $\quad$ preserves mirror symmetry in the stacking direction around one layer [28] with the reflection operator that acquires an explicit momentum dependence and reads $\mathcal{M}\left(k_{z}\right)=\operatorname{diag}\left(1, e^{-i k_{z}}\right)$. The mirror symmetry constraint $\mathcal{M}\left(k_{z}\right) \mathcal{H}_{S} \mathcal{M}\left(k_{z}\right)^{-1} \equiv$ $\mathcal{H}_{S}\left(k_{z} \rightarrow-k_{z}\right)$ implies a decoupling of the chiral modes on the $k_{z}=\pi$ line. Hence, the three-dimensional system is characterized by gapless surfaces with a mirror-symmetry protected single Dirac cone with the Dirac point at $\left\{k_{x}, k_{z}\right\}=\{0, \pi\}$.

The presence of this single surface Dirac cone can also be seen as a consequence of the fact that the bulk threedimensional Hamiltonian is characterized by a nonzero mirror Chern number [32] $\mathcal{C}_{\mathcal{M}}=1$ at $k_{z}=\pi$. When considering systems with a finite number of layers still preserving reflection symmetry in the stacking direction-this constraint is fulfilled for stacks with an odd number of layers - the surface spectrum in the remaining translational invariant $k_{x}$ direction will therefore display a single uncoupled chiral mode as schematically shown in Fig. 1(b). We emphasize that this chiral anomaly is regularized by the presence of a chiral mode partner with opposite chirality at the opposite (010) surface.

We next "trivialize" our system by introducing a perturbation that explicitly breaks the mirror symmetry along the stacking direction. To this end, we consider a dimerization pattern in the interlayer coupling which further modifies the effective surface Hamiltonian as $\mathcal{H}_{S}=\mathcal{H}_{0}+$ $\left[t_{1}+t_{2} \cos \left(k_{z}\right)\right] \sigma_{x}-t_{2} \sin \left(k_{z}\right) \sigma_{y}$ [cf. Fig. 1(a)]. This, in turn, implies that the single surface Dirac cone acquires a mass $\propto t_{1}-t_{2}$ and the surface becomes a conventional gapped one. When considering, as before, a finite system with an odd number of layers there will be a single chiral mode traversing the full surface gap [cf. Fig. 1(c)] localized at one of the two boundary layers depending upon the specific dimerization pattern. The existence of this chiral hinge mode can be understood by considering the effective surface Hamiltonian as a collection of one-dimensional Rice-Mele models [33,34], parametrized by $k_{x}$. For a chain with an odd number of sites, the latter displays an in-gap boundary state at an energy corresponding precisely to the staggered chemical potential $\equiv k_{x}$. If we assume the dimerization pattern to be equivalent at all four surfaces perpendicular to the layer planes, the hinge modes at the boundary layer will be connected to create a circulating planar current, which is in agreement with the fact that a dimerized stack of an odd number of layers defines a (thicker) two-dimensional Chern insulating state [35].

Let us now instead assume that the dimerization patterns at the two opposite surfaces (010) and $(0 \overline{1} 0)$ are designed to be opposite to each other as shown in Fig. 1(a). Although still breaking mirror symmetry along the stacking direction, this configuration preserves bulk inversion symmetry with the inversion center lying at the center of one layer. The presence of inversion symmetry also implies that the chiral hinge modes related to the (010) and (010) surfaces will be localized on opposite boundary layers. The same clearly holds true at the (100) and (100) surfaces. Moreover, the Jackiw-Rebbi mechanism [36] guarantees the existence of an additional chiral hinge mode at two inversion-symmetry related hinges between the $x$ and $y$ planes, and thus the configuration of ingap hinge states schematically shown in Fig. 1(c) is realized. The latter represents nothing but the hallmark of a secondorder topological insulator in three dimensions (3D) protected by inversion symmetry.

\section{QUANTUM ANOMALOUS HALL STACKS}

Having presented our coupled-layer low-energy approach, we next introduce a microscopic model that features an inversion-symmetric higher-order topological insulating state. Specifically, we consider stacks of honeycomb layers, each of which possesses chiral orbital currents leading to a quantum anomalous Hall (QAH) insulating state [29]. In order to have alternating Chern numbers on the honeycomb layers, we further assume the direction of the orbital currents to be opposite in two consecutive layers. For uncoupled layers, the corresponding tight-binding Hamiltonian reads

$$
\mathcal{H}_{\|}=-t \sum_{\langle i, j\rangle, \alpha} c_{i \alpha}^{\dagger} c_{j \alpha}-i t_{2} \sum_{\langle\langle i, j\rangle\rangle, \alpha}(-1)^{\alpha} v_{i j} c_{i \alpha}^{\dagger} c_{j \alpha},
$$

where $c_{i \alpha}^{\dagger}\left(c_{i \alpha}\right)$ creates (annihilates) an electron on site $i$ in layer $\alpha, t$ is the intralayer nearest-neighbor hopping amplitude, and $t_{2}$ is the next-nearest-neighbor hopping amplitude. As usual, the factor $v_{i j}=1$ if the next-nearest-neighbor hopping path rotates counterclockwise, and -1 if it rotates clockwise. We next introduce an interlayer coupling that explicitly breaks the mirror symmetry in the stacking direction but still preserves bulk inversion symmetry with the inversion center in one layer at the center of the bond between the two $A$ and $B$ honeycomb sublattices. In its simplest form the interlayer Hamiltonian is then

$$
\begin{aligned}
\mathcal{H}_{\perp}= & -\frac{t_{z}}{2} \sum_{i \in A, \alpha}\left[1-e^{i \pi \alpha}\right] c_{i \alpha}^{\dagger} c_{i \alpha+1}-\frac{t_{z}}{2} \sum_{i \in B, \alpha}\left[1+e^{i \pi \alpha}\right] \\
& \times c_{i \alpha}^{\dagger} c_{i \alpha+1}+\text { H.c. }
\end{aligned}
$$

As discussed in the Supplemental Material [37], this interlayer Hamiltonian is naturally realized assuming buckled honeycomb layers. Figure 2(a) shows the edge energy spectrum as obtained by diagonalizing the full Hamiltonian $\mathcal{H}=\mathcal{H}_{\|}+$ $\mathcal{H}_{\perp}$ for stacks of zigzag terminated ribbons with an odd number of layers. It agrees perfectly with the foregoing lowenergy description. Inside the gapped bulk energy bands, we 

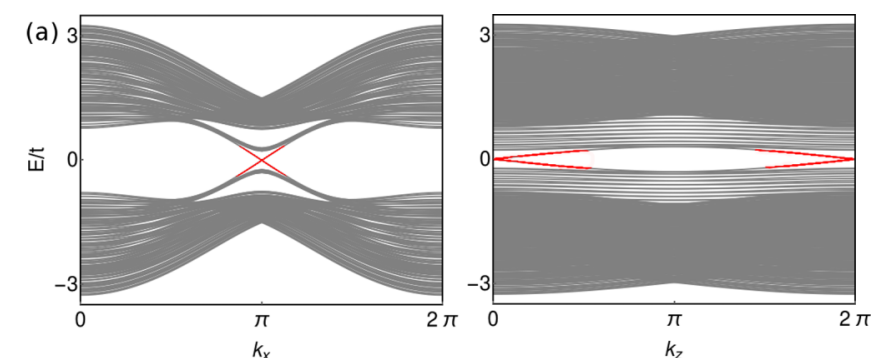

(b)

$L_{3}$

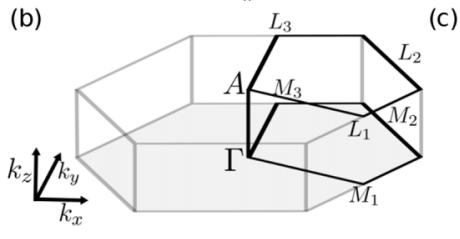

(c)

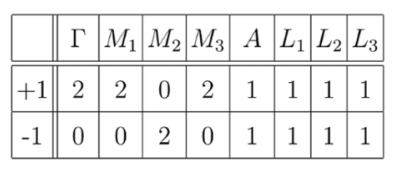

FIG. 2. (a) Edge spectra of the QAH model with periodic boundary conditions in the $x$ and $z$ direction for stacks of zigzag-terminated honeycomb flakes. The tight-binding Hamiltonian parameters have been chosen as $t_{2} / t=0.2$ and $t_{z} / t=0.3$. (b) The hexagonal 3D Brillouin zone with the inversion symmetric points labeled. (c) Table specifying the number of bands with inversion eigenvalue +1 and -1 at the inversion symmetric points in the Brillouin zone.

clearly observe conventional surface states, corresponding to a massive surface Dirac cone, in the gap of which two chiral hinge modes localized on opposite layers appear. Precisely the same features occur considering periodic boundary conditions in the stacking direction and zigzag terminations in the other two directions. We point out that we excluded from our analysis ribbons with armchair terminations since the latter would yield an unprotected single massless surface Dirac cone. This is due to the fundamental difference between the chiral edge states of a QAH insulator for zigzag and armchair terminated ribbons [38]. For the latter, the chiral edge states have an equal amplitude on both the two honeycomb sublattices. The interlayer coupling Hamiltonian introduced above thus yields an effective mirror-symmetric coupling between the QAH chiral edge states. However, additional symmetry-allowed terms in the bulk Hamiltonian, e.g., intralayer real next-nearestneighbor hopping amplitudes, will gap the surface Dirac cone leading to the observation of chiral hinge modes even for stacks of armchair terminated ribbons.

To prove the topological origin of these chiral hinge states, we have calculated the bulk $\mathbb{Z}_{2}$ topological invariant for a second-order topological insulator with inversion symmetry [25]. It can be derived using the bulk formulation of the quantized corner charges for the effective two-dimensional inversion-symmetric Hamiltonians at $k_{z}=0, \pi$, and thereafter considering the corresponding corner charge flow. When expressing the corner charges in terms of the multiplicities of the inversion-symmetry eigenvalues \pm 1 of the occupied bands at the inversion-symmetric momenta of the 3D Brillouin zone [cf. Fig. 2(b)], we then find the following expression for the bulk $\mathbb{Z}_{2}$ invariant:

$$
\begin{aligned}
v= & {\left[-\Gamma_{1}-\frac{1}{2} \Gamma_{-1}+\frac{1}{2}\left[\left(M_{1}\right)_{-1}+\left(M_{2}\right)_{-1}+\left(M_{3}\right)_{-1}\right]\right.} \\
& \left.+A_{1}+\frac{1}{2} A_{-1}-\frac{1}{2}\left[\left(L_{1}\right)_{-1}+\left(L_{2}\right)_{-1}+\left(L_{3}\right)_{-1}\right]\right] .
\end{aligned}
$$

A nontrivial value $v=1 \bmod 2$ of this invariant guarantees the presence of chiral hinge modes provided the bulk and

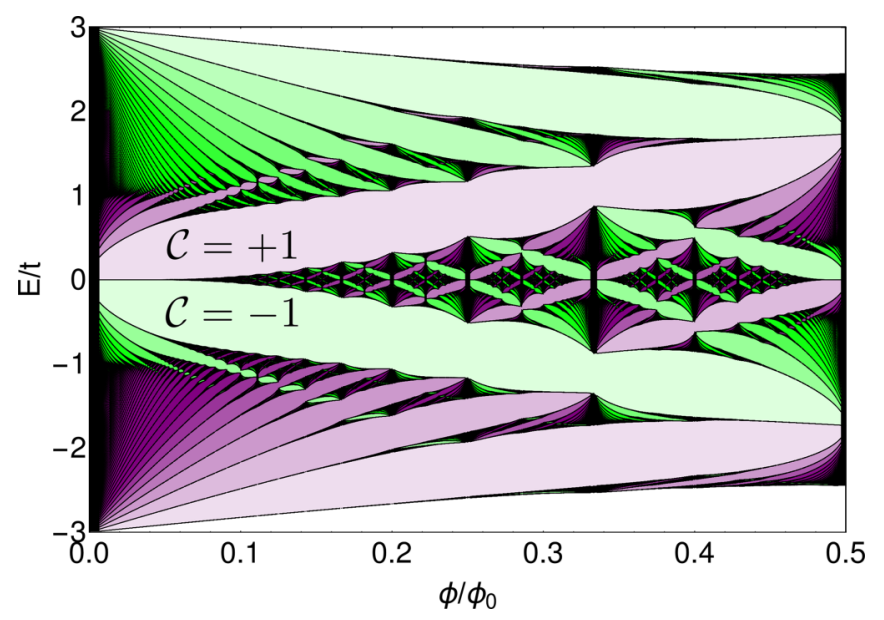

FIG. 3. Energy versus magnetic flux $\phi$ (measured in units of the magnetic flux quantum $\phi_{0}$ ) for the Hofstadter model on a honeycomb lattice. The gaps are colored according to their Chern number. The two largest gaps have $\mathcal{C}= \pm 1$.

the surfaces are gapped. For our model at half-filling, the inversion-symmetry labels [cf. Fig. 2(c)] directly imply an higher-order topology. Therefore the chiral hinge states shown in Fig. 2(a) are the direct manifestation of a bulk-hinge correspondence.

\section{STACKS OF QUANTUM HALL LAYERS}

With these results in hand, we next introduce our main result: the possibility to engineer an inversion-symmetry protected second-order topological insulator in stacks of doped quantum Hall layers with a honeycomb lattice structure. To show this, we first recall that the quantum Hall effect on the honeycomb lattice exhibits a zeroth Landau level above (below) which the total Chern number $\mathcal{C}=+1(-1)$ for relatively weak magnetic fluxes per plaquette (see Fig. 3). This also implies that the sign of the Hall conductance is opposite in $p$ and $n$-doped layers. We then take advantage of this property to realize a quantum Hall analog of the quantum anomalous Hall model introduced above. For the intralayer part of the Hamiltonian we thus consider layers with an alternating bias $\pm V_{0}$. The corresponding Hamiltonian is then

$$
\mathcal{H}_{\|}=-t \sum_{\langle i, j\rangle, \alpha} c_{i, \alpha}^{\dagger} c_{j, \alpha}+V_{0} \sum_{i, \alpha}(-1)^{\alpha} c_{i, \alpha}^{\dagger} c_{i, \alpha} .
$$

The effect of the perpendicular magnetic field is taken into account via the usual Peierls substitution $t \rightarrow t e^{i e \int d \mathbf{s} \cdot \mathbf{A} / \hbar}$, where $d \mathbf{s}$ is the line integral between the bonds, $e$ is the electron charge, and we took the vector potential in the Landau gauge $\mathbf{A}=(-B y, 0,0)$ with $B$ the magnetic field strength. The interlayer Hamiltonian can be taken to be the same as in Eq. (1) when considering the buckled honeycomb layers realized in silicene [39]. However, to describe stacks of graphene layers we will consider a mirror-symmetric interlayer Hamiltonian of the form $\mathcal{H}_{\perp}=-t_{z} \sum_{\alpha} c_{i, \alpha}^{\dagger} c_{i, \alpha+1}+$ H.c. [cf. Fig. 4(a)].

Since the external perpendicular magnetic field does not break the mirror symmetry in the stacking direction, we 

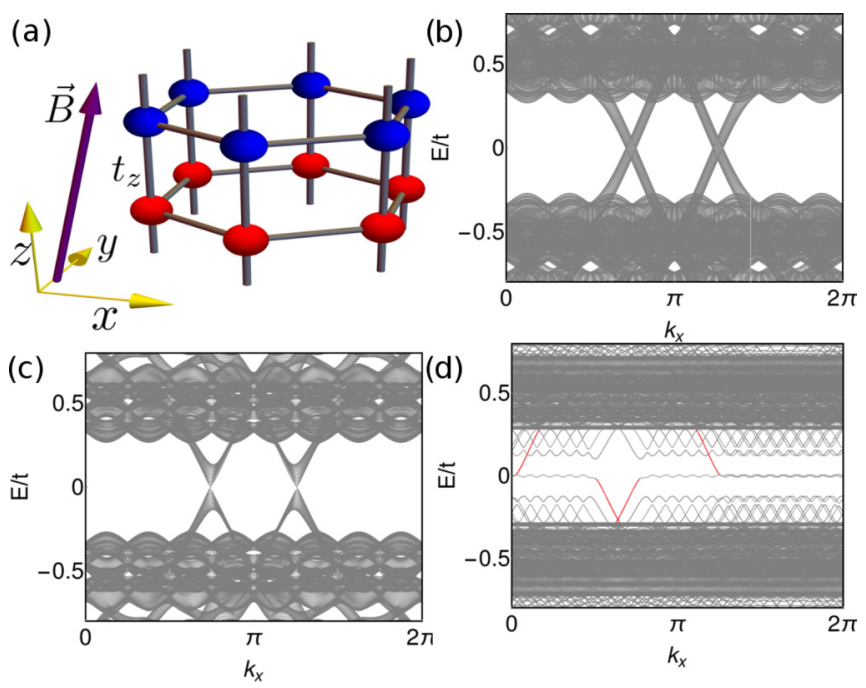

FIG. 4. (a) Schematic side view of an AA stacked honeycomb lattice in a canted magnetic field. (b) Spectrum for a zigzag ribbon of alternately doped stacks of graphene with $t / t_{z}=0.2, \phi / \phi_{0}=1 / 8$, $V_{0}=0.5$, and $\phi_{2} / \phi_{0}=\phi_{3} / \phi_{0}=0$, where $\phi / \phi_{0}, \phi / \phi_{1}$, and $\phi / \phi_{2}$ are the flux per plaquette in the $z, x$, and $y$ direction, respectively. (c) Same as (b), but now with a magnetic field in the $x$ direction $\phi_{2} / \phi_{0}=$ 0.05 . (d) Same as (b), but now with a magnetic field in the $x$ direction $\phi_{2} / \phi_{0}=0.05$, and $y$ direction $\phi_{3} / \phi_{0}=0.01$.

expect the presence of protected surface Dirac cones inside the bulk band gap $\propto V_{0}$. This is verified in Fig. 4(b) where we show the energy spectrum for stacks of zigzag terminated ribbons with an odd number of layers. Note that due to the remaining chiral symmetry of our microscopic tight-binding model the surface energy spectrum features Dirac nodal lines, which underline the absence of an effective interlayer coupling between the quantum Hall edge states.

In order to drive the system from a mirror Chern insulating phase to a second-order topological insulating phase, we next introduce an additional component of the magnetic field in the layer plane, which breaks the mirror symmetry in the stacking direction but preserves the bulk inversion symmetry of our AA-stacked graphene multilayers. Figure 4(c) shows the edge spectrum when superimposing a finite component of the magnetic field parallel to the zigzag direction. The nodal degeneracies are removed but the surface still features an unprotected surface Dirac cone signaling a coupling between the quantum Hall edge states that is still effectively mirror symmetric. We get rid of this additional unprotected degeneracy by further adding a magnetic field component in the layer plane but perpendicular to the zigzag direction. This leads to surface gap openings with the edge spectrum [cf. Fig. 4(d)] that is now composed of surface Landau levels and additionally features chiral states localized at opposite hinges. Moreover, the chiral hinge states at positive and negative energies are separated by a zeroth surface Landau level, and therefore live on different
TABLE I. Inversion eigenvalues at half-filling at inversionsymmetric points in the Brillouin zone for $\phi / \phi_{0}=1 / 3, V_{0}=0.8 t$.

\begin{tabular}{ccccccccc}
\hline \hline & $\Gamma$ & $M_{1}$ & $M_{2}$ & $M_{3}$ & $A$ & $L_{1}$ & $L_{2}$ & $L_{3}$ \\
\hline+1 & 5 & 3 & 3 & 3 & 3 & 3 & 3 & 3 \\
-1 & 1 & 3 & 3 & 3 & 3 & 3 & 3 & 3 \\
\hline \hline
\end{tabular}

hinges (see the Supplemental Material [37]). As discussed above, this change in the location of the chiral hinge modes by tuning the Fermi level is perfectly compatible with the topology of our system.

Finally, to verify the bulk nontrivial topology of our microscopic tight-binding model, we have computed the multiplicities of the inversion-symmetry eigenvalues at the inversionsymmetric momenta of the BZ (see Table I). When computing Eq. (2) we then find a nontrivial value of the $\mathbb{Z}_{2}$ topological invariant in perfect agreement with the foregoing analysis. As shown in the Supplemental Material [37], the existence of an inversion-symmetric second-order topological insulator in stacks of doped quantum Hall layers is not restricted to graphene layers but can be also extended to buckled structures, e.g., silicene, and even multilayer phosphorene [40], thus strengthening the generality of our design principle.

\section{CONCLUSION}

To sum up, we have proved the existence of a secondorder topological insulator protected by inversion symmetry using a coupled layer approach in which the layers have alternating Chern numbers. It can be derived from a parent topological mirror Chern insulator by crystalline symmetry breaking terms that retain the bulk inversion symmetry of the bulk crystal. The presence of the topologically protected chiral hinge modes can be inferred from a three-dimensional $\mathbb{Z}_{2}$ invariant. We have shown that a nontrivial value of this invariant can be encountered in stacks of doped honeycomb layers subject to an external magnetic field slightly tilted away from the stacking direction. The required alternating doping of the honeycomb sheets can be obtained for instance by separating the monolayers with two-dimensional polar spacers [41]. As a result, we believe that graphene, silicene, and phosphorene multilayers provide an excellent platform to engineer chiral inversion-symmetric higher-order topological insulators.

\section{ACKNOWLEDGMENTS}

C.O. acknowledges support from a VIDI Grant (Project 680-47-543) financed by the Netherlands Organization for Scientific Research (NWO). This work is part of the research programme of the Foundation for Fundamental Research on Matter (FOM), which is part of the Netherlands Organization for Scientific Research (NWO). S.K. acknowledges support from a NWO-Graduate Program grant.
[1] B. Bradlyn, L. Elcoro, J. Cano, M. G. Vergniory, Z. Wang, C. Felser, M. I. Aroyo, and B. A. Bernevig, Nature (London) 547, 298 (2017).
[2] H. C. Po, A. Vishwanath, and H. Watanabe, Nat. Commun. 8, 50 (2017).

[3] M. Z. Hasan and C. L. Kane, Rev. Mod. Phys. 82, 3045 (2010). 
[4] X.-L. Qi and S.-C. Zhang, Rev. Mod. Phys. 83, 1057 (2011).

[5] L. Fu, Phys. Rev. Lett. 106, 106802 (2011).

[6] T. H. Hsieh, H. Lin, J. Liu, W. Duan, A. Bansil, and L. Fu, Nat. Commun. 3, 982 (2012).

[7] H. Nielsen and M. Ninomiya, Phys. Lett. B 105, 219 (1981).

[8] C. Fang and L. Fu, arXiv:1709.01929.

[9] W. A. Benalcazar, B. A. Bernevig, and T. L. Hughes, Science 357, 61 (2017).

[10] W. A. Benalcazar, B. A. Bernevig, and T. L. Hughes, Phys. Rev. B 96, 245115 (2017).

[11] F. Schindler, A. M. Cook, M. G. Vergniory, Z. Wang, S. S. Parkin, B. A. Bernevig, and T. Neupert, Sci. Adv. 4, eaat0346 (2018)

[12] F. Schindler, Z. Wang, M. G. Vergniory, A. M. Cook, A. Murani, S. Sengupta, A. Y. Kasumov, R. Deblock, S. Jeon, I. Drozdov et al., Nat. Phys. 14, 918 (2018).

[13] M. Geier, L. Trifunovic, M. Hoskam, and P. W. Brouwer, Phys. Rev. B 97, 205135 (2018).

[14] Y. Xu, R. Xue, and S. Wan, arXiv:1711.09202.

[15] C. W. Peterson, W. A. Benalcazar, T. L. Hughes, and G. Bahl, Nature (London) 555, 346 (2018).

[16] M. Serra-Garcia, V. Peri, R. Süsstrunk, O. R. Bilal, T. Larsen, L. G. Villanueva, and S. D. Huber, Nature (London) 555, 342 (2018).

[17] S. Imhof, C. Berger, F. Bayer, J. Brehm, L. W. Molenkamp, T. Kiessling, F. Schindler, C. H. Lee, M. Greiter, T. Neupert et al., Nat. Phys. 14, 925 (2018).

[18] E. Khalaf, Phys. Rev. B 97, 205136 (2018).

[19] M. Ezawa, Phys. Rev. Lett. 120, 026801 (2018).

[20] J. Langbehn, Y. Peng, L. Trifunovic, F. von Oppen, and P. W. Brouwer, Phys. Rev. Lett. 119, 246401 (2017).

[21] M. Sitte, A. Rosch, E. Altman, and L. Fritz, Phys. Rev. Lett. 108, 126807 (2012).
[22] Z. Song, Z. Fang, and C. Fang, Phys. Rev. Lett. 119, 246402 (2017).

[23] M. Ezawa, Phys. Rev. B 97, 155305 (2018).

[24] M. Ezawa, Phys. Rev. B 97, 241402 (2018).

[25] G. van Miert and C. Ortix, Phys. Rev. B 98, 081110 (2018).

[26] A. Lau, K. Koepernik, J. van den Brink, and C. Ortix, Phys. Rev. Lett. 119, 076801 (2017).

[27] A. C. Neto, F. Guinea, N. M. Peres, K. S. Novoselov, and A. K. Geim, Rev. Mod. Phys. 81, 109 (2009).

[28] I. C. Fulga, N. Avraham, H. Beidenkopf, and A. Stern, Phys. Rev. B 94, 125405 (2016).

[29] F. D. M. Haldane, Phys. Rev. Lett. 61, 2015 (1988).

[30] D. R. Hofstadter, Phys. Rev. B 14, 2239 (1976).

[31] B. A. Bernevig, T. L. Hughes, S. Raghu, and D. P. Arovas, Phys. Rev. Lett. 99, 146804 (2007).

[32] J. C. Y. Teo, L. Fu, and C. L. Kane, Phys. Rev. B 78, 045426 (2008).

[33] M. J. Rice and E. J. Mele, Phys. Rev. Lett. 49, 1455 (1982).

[34] D. Xiao, M.-C. Chang, and Q. Niu, Rev. Mod. Phys. 82, 1959 (2010).

[35] L. Fu and C. L. Kane, Phys. Rev. B 76, 045302 (2007).

[36] R. Jackiw and C. Rebbi, Phys. Rev. D 13, 3398 (1976).

[37] See Supplemental Material at http://link.aps.org/supplemental/ 10.1103/PhysRevB.98.245102 for a detailed description of the localization of the hinge states and additional calculations for silicene and phosphorene.

[38] L. Cano-Cortés, C. Ortix, and J. van den Brink, Phys. Rev. Lett. 111, 146801 (2013).

[39] C. Kamal, A. Chakrabarti, A. Banerjee, and S. Deb, J. Phys.: Condens. Matter 25, 085508 (2013).

[40] A. N. Rudenko, S. Yuan, and M. I. Katsnelson, Phys. Rev. B 92, 085419 (2015).

[41] S. K. Das, B. Yan, J. van den Brink, and I. C. Fulga, arXiv:1807.10078. 